\title{
Isolated Mediastinal Kocuria rosea Infection Mimicking Malignancies
}

\author{
Alexander Emmert ${ }^{\mathrm{a}, \mathrm{e}}$, Franke Robert ${ }^{\mathrm{a}}$, Hanibal Bohnenberger ${ }^{\mathrm{b}}$, Carsten-Oliver Sahlmann ${ }^{\mathrm{c}}$, \\ Friedrich A. Schondube ${ }^{a}$, Michael Egan ${ }^{\mathrm{d}}$, Theodor Tirilomis ${ }^{\mathrm{a}}$, Martin Friedrich ${ }^{\mathrm{a}}$, \\ Bernhard C. Danner ${ }^{a}$, Marc Hinterthaner ${ }^{\mathrm{a}}$
}

\begin{abstract}
Isolated mediastinal Kocuria rosea infection is a rare condition and presentation can be variable. We report an unusual case of Kocuria rosea infection which presented as solitary pulmonary nodules and isolated lymph node swelling, entirely confined within the tracheal bifurcation and 18-fluoredeoxyglucose position emission tomography and computed tomography (18FDG PET/CT)-avid, thereby mimicking a neoplastic lesion. Owing to the large differential diagnosis, which includes diseases such as pulmonary neoplasia and lymph node metastases, a biopsy via mediastinoscopy was to be attempted, so hopefully avoiding thoracotomy. Histopathology analysis of the resected mediastinal lymph nodes (MLNs) demonstrated no malignant cells, but rather necrotizing MLNs, which is classically associated with Kocuria rosea infection. The patient was asymptomatic and biopsy allowed a precise diagnosis. Kocuria rosea infection is rare; it should be considered when FDG PET shows intense FDG uptake in non-regionally swollen lymph nodes.
\end{abstract}

Keywords: Mediastinal lymph nodes; Mediastinoscopy; Kocuria rosea

\section{Introduction}

Enlarged lymph nodes in the mediastinum can be due to neo-

Manuscript accepted for publication June 02, 2015

aDepartment of Thoracic and Cardiovascular Surgery, University of Gottingen, Robert-Koch-Str. 40, 37075 Gottingen, Germany

${ }^{b}$ Department of Pathology, University of Gottingen, Robert-Koch-Str. 40, 37075 Gottingen, Germany

'Department of Nuclear Medicine, University of Gottingen, Robert-Koch-Str. 40, 37075 Gottingen, Germany

dDepartment of Anaesthesiology, University of Gottingen, Robert-Koch-Str. 40, 37075 Gottingen, Germany

${ }^{\mathrm{e}}$ Corresponding Author: Alexander Emmert, Department of Thoracic and Cardiovascular Surgery, University Medical Center, Georg-August University, Robert-Koch-Str. 40, D-37075, Gottingen, Germany.

Email: alexander.emmert@med.uni-goettingen.de

doi: http://dx.doi.org/10.14740/jmc2122w plasia infection or other diseases and the classification of these nodes is crucial to correct management of such a patient [1]. Advances in imaging of mediastinal lymph nodes (MLNs) with 18-fluoredeoxyglucose position emission tomography and computed tomography (18FDG PET/CT) have improved diagnostic accuracy and determination of nodal status. However, due to the low specificity of PET positive MLNs should be biopsied to confirm or rule out metastasis and invasive surgical diagnostic techniques such as mediastinoscopy are the preferred biopsy methods of obtaining MLN tissue.

Kocuria rosea, an aerobic Gram-positive coccus is generally regarded as a non-pathogenic commensal that colonizes the oropharynx, skin, and mucosa. Nonetheless, it is capable of causing opportunistic infection in immunocompromised patients [2].

We report the case of PET positive, necrotizing MLN associated with Kocuria rosea in an otherwise healthy young man. Only a limited number of Kocuria rosea infections are reported in the literature and ours seems to be only the second published case and the first in which diagnosis and treatment have been carried out by mediastinoscopy.

\section{Case Report}

We report the case of a young adult male who presented with mediastinal lymphadenitis in the absence of commonly associated features: no compromised immunity or HIV-infection, no signs or symptoms of infection or mediastinum involvement, and a rapid response to therapy. In addition, the patient reported no symptoms of upper respiratory tract infection, had no palpable lymph nodes or no fever. Additionally, all laboratory results, including blood count, biochemical findings, and C-reactive protein, were within normal range. A PET/CT was performed, and abnormal 18FDG uptake was apparent around the area of tracheal bifurcation. The standardized uptake value (SUV max) was 8.9 (Fig. 1). A mediastinal lymphadenectomy was performed, and histological lymph node analysis confirmed necrotizing lymphadenitis. Microbiological diagnosis of the tissue was performed and culture positive for Kocuria rosea was obtained. Antibiotic sensitivity tests were not performed and 3 days after admission, the patient was discharged from the hospital in good condition. 


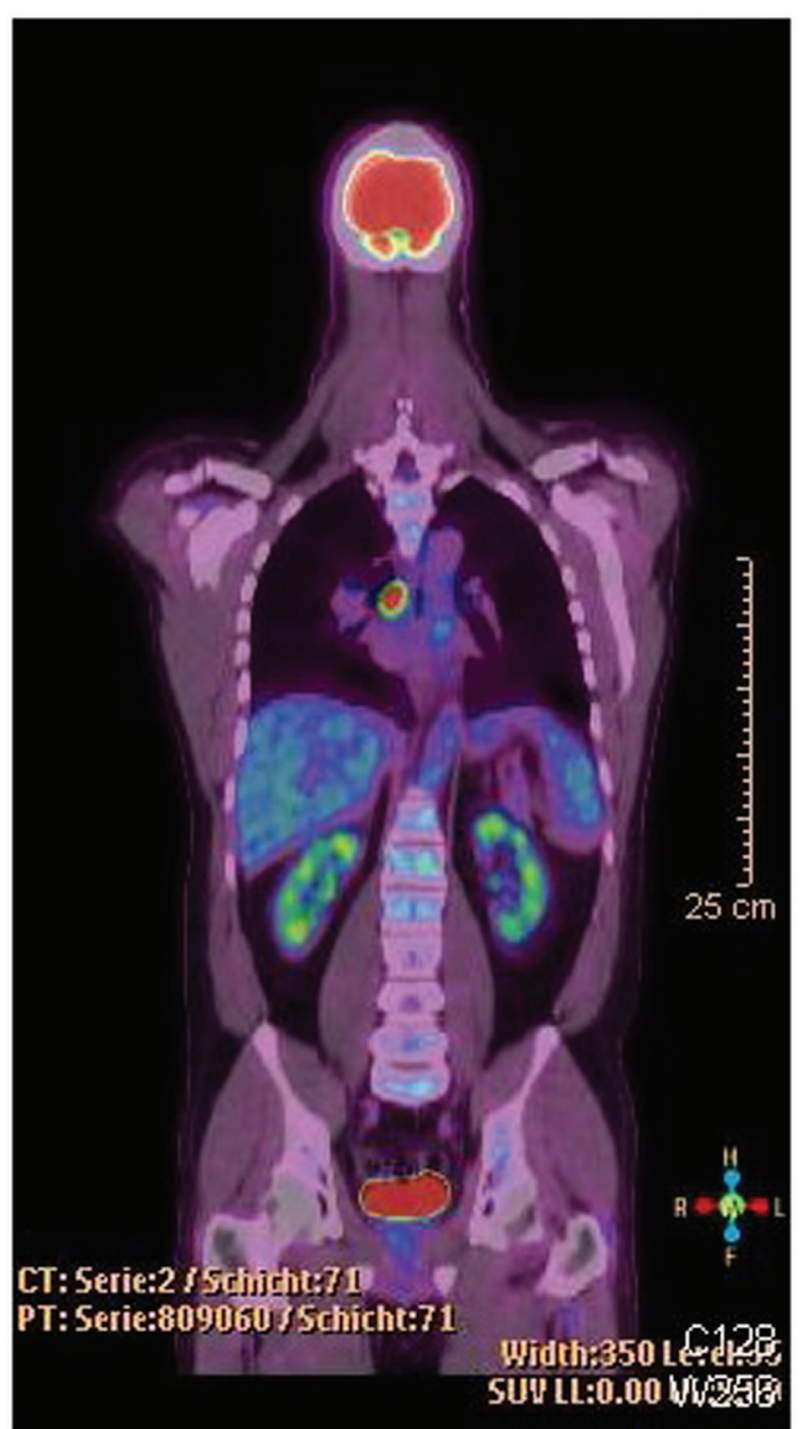

Figure 1. PET/CT showed a swollen mediastinal lymph node measuring $10 \mathrm{~mm}$ in diameter at the tracheal bifurcation.

\section{Discussion}

Kocuria is a member of the Micrococcaceae family, which presently comprises 18 species [3]. Although generally regarded as harmless saprophytes that inhabit or colonize skin and oropharynx mucosa this aerobic, non-encapsulated, nonmotile bacteria can cause opportunistic infection in the immunocompromised host [3]. Infections such as endocarditis, arthritis, central nervous system infection, pneumonia, peritonitis, hepatic abscess and nosocomial blood stream infections have all being reported [2-4]. In addition, strains identified as Micrococcus spp. have been reported recently in infections associated with indwelling intravenous lines, continuous ambulatory peritoneal dialysis fluids, ventricular shunts and prothetic valves [2-5]. Our case of Kocuria infection is interesting as its unusual presentation made the diagnosis of Kocuria rosea infection very difficult to consider at all.
18FDG PET/CT scanning has been useful in the evaluation of malignant disorders and has been extensively used in cancer screening. However, 18 FDG uptake is not specific to cancerous lesions, but is also present in more benign diseases such as infection and inflammation, particularly granulomatous diseases [6]. However, occasionally, a granulomatous isolated necrotizing lymph node presents focal "hot spots", which can be easily misinterpreted as malignancy. This report emphasizes that granulomatous necrotizing lymph nodes can present a potential pitfall when interpreting FDG scans.

\section{Conclusion}

Kocuria rosea infection is rare; it should be considered when FDG PET shows intense FDG uptake in non-regionally swollen lymph nodes.

\section{Acknowledgement}

We acknowledge support by the German Research Foundation and the Open Access Publication Funds of the Gottingen University.

\section{Competing Interests}

The authors declare that they have no competing interests.

\section{Abbreviations}

18FDG PET/CT: 18-fluoredeoxyglucose position emission tomography and computed tomography; MLN: mediastinal lymph node; SUV: standardized uptake value

\section{References}

1. Hocke M, Menges M, Topalidis T, Dietrich CF, Stallmach A. Contrast-enhanced endoscopic ultrasound in discrimination between benign and malignant mediastinal and abdominal lymph nodes. J Cancer Res Clin Oncol. 2008;134(4):473-480.

2. Lee MK, Choi SH, Ryu DW. Descending necrotizing Mediastinitis caused by Kocuria rosea: a case report. BMC Infect Dis. 2013;13:475.

3. Ma ES, Wong CL, Lai KT, Chan EC, Yam WC, Chan AC. Kocuria kristinae infection associated with acute cholecystitis. BMC Infect Dis. 2005;5:60.

4. Bannerman TL. Staphylococcus, Micrococcus and other catalase-positive cocco that grow aerobically, Chapter 28. In: Murray PR, Baron EJ, Jorgensen JH, Eds. Manual of clinical Microbiology, 8th ed. Washington DC: ASM Press. 2003;I:385-404.

5. Peces R, Gago E, Tejada F, Laures AS, Alvarez-Grande J. Relapsing bacteraemia due to Micrococcus luteus in a 
haemodialysis patient with a Perm-Cath catheter. Nephrol Dial Transplant. 1997;12(11):2428-2429.

6. Park WJ, Kim EK, Park JH. Extensive tuberculous lym- phadenitis mimicking distant lymph node metastasis on F-18FDG PET/CT in a patient with a history of malignant melanoma. Yonsei Med J. 2013;54(6):1554-1556 